\title{
An improved algorithm for the Cellular Element Method
}

\author{
Liu Jie Yang Jixin Yang Jingnan \\ School of Transportation, Wuhan University of Technology, Wuhan, China \\ liujie6699@vip.163.com,whutvses@163.com,yin870413@163.com
}

\begin{abstract}
Key words: cellular element method, basic algorithm, improved algorithm, computing speed Abstract: A cellular element method turns the global analysis of the finite element method into a series of partial analyses. The final overall balance is achieved through the partial unbalanced transfer of force. The requirement for computer memory is extremely low and parallel algorithms are easy to implement. However, the computing speed is slower than that of the finite element method. To solve this problem, an improved algorithm was put forward and the basic principle was described. The detailed calculation steps were listed and five different density grid models were given as examples. The computing time of the two algorithms were compared and the results show that the computing speed by the improved algorithm was increased significantly.
\end{abstract}

\section{Introduction}

The finite element method has a lot of advantages such as strong adaptability, simple concept, easy to learn and easy to program. It has been widely used in solving practical engineering problems. However, finite element method does not reach the level of perfection. Two problems have cost researchers a great deal of time and effort in the past decades. One problem was the storage of the global stiffness matrices and mass matrices take up a lot of memory so that computing scale was limited by the capacity of the machine. Large scale structures cannot be calculated on a personal computer, and extra-large structures were even difficult to analyze on a mainframe or workstation. The second problem was the parallel computation of the finite element method ${ }^{[1-3]}$. Since the advent of parallel computers in the early 1970s, many researchers worked hard for parallel algorithms of finite element analysis, opened up the new field of parallel computational mechanics and explored various strategies and techniques for improving parallelism. However, the results were still unsatisfactory. To solve the problems, Shen Chengwu, Yang Jixin $^{[4-5]}$ et al, developed a new method - the cellular element method. It turns the global analysis of structure into a series of partial analyses.

The final overall balance is achieved through the partial unbalanced transfer of force. The requirement for computer memory is extremely low and parallel algorithms are easy to implement. The accuracy of the cellular element method has been verified through the calculation of some structures. However, the computing speed is slower than the finite element method. So, how to improve the computing speed or reduce the computing time is a problem worthy of study. This paper presents an improved algorithm.

\section{Principle of the Cellular Element Method}

For the reason that cellular element method is not well known yet, a brief introduction of its basic principle is provided as follows:

For a structure which needs to be studied, it is divided into nodes and elements in the same way as the finite element method. A node and its neighbor nodes and elements form a cell. The cell whose center node is $N_{i}$ defined as $D_{i}$. $D_{i}$ can be expressed in terms of $m_{i}$ elements $E_{i}^{j}$, $n_{i}$ neighbor nodes $N_{i}^{k}$ :

$$
\overline{\bar{i}} \quad D_{i} \cup\left\{N_{i}^{1} \cdots ; E,,_{i}{ }_{i} ; E{ }_{i}^{1} \cdot N,{ }_{i}^{n_{i}} N \quad{ }_{i} N \in{ }_{i}^{j} E \quad N \in E \quad j=1 \cdots, m_{i}\right\}
$$

where, cell $D_{i}$ includes node $N_{i}$, the neighbor element $E_{i}^{j}\left(j=1, \ldots, m_{i}\right)$ and nodes $N_{i}^{k}\left(k=1, \ldots, n_{i}\right)$.

The mechanical characteristic of cell $D_{i}$ can be expressed as

$$
V_{i}=\left\{u_{p} F_{i}\right\}
$$


where $u_{i}$ and $F_{i}$ are the generalized displacement and generalized nodal force of node $N_{i}$. The dimension of $V_{i}$ is $2 \mathrm{R}$, it varies due to different structures. It can be $R=2$ (plane truss, plane problem in elasticity mechanics), $R=3$ (plane frame, plate, three-dimensional entity) or $R=6$ (space frame, shell).

A cellular model for solving a plane stress problem is shown in Fig.1 and Fig.2 as an example.

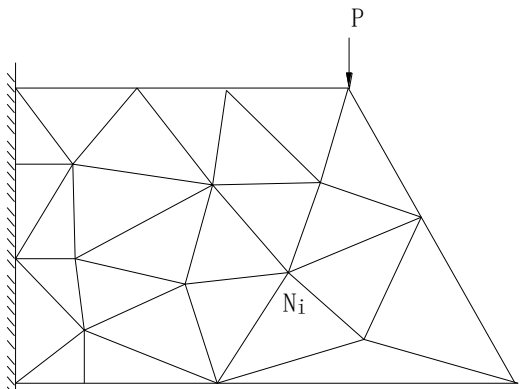

Fig.1 Discrete plane structure

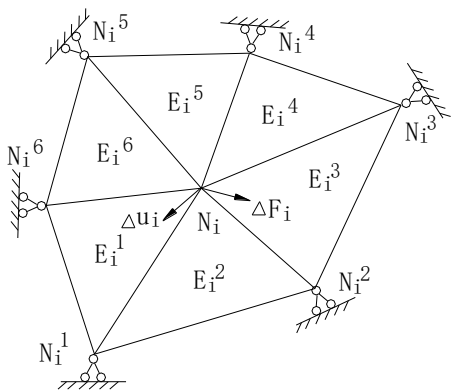

Fig.2 Cellular model

The structure in Fig. 1 is divided into $e$ elements and $n$ nodes in the same way as the finite element method. For the sake of simplicity, the three-node constant strain triangular element is used here.

For any nodes $N_{i}(\mathrm{i}=1,2, \ldots \mathrm{n})$, give the following definition:

(1) Neighbor element of a node: If $N_{i}$ is a node of element $E$, then $E$ is called a neighbor element of node $N_{i}$. Neighbor element is also called related element, $N_{i}$ has 6 neighbor elements $E_{i}^{j}(\mathrm{j}=1,2, \ldots 6)$ in Fig.2.

(2) Neighbor node of a node. If a node $N$ and $N_{i}$ belong to the same element, then $N$ is called a neighbor node of node $N_{i}$. Neighbor node is also called related node. $N_{i}$ has 6 neighbor nodes $N_{i}^{k}$ $(\mathrm{k}=1,2, \ldots 6)$ in Fig.2.

(3) Cell: Taking the node $N_{i}$ as the center, the lattice system composed of $N_{i}$ and its neighbor elements and nodes is called a cell, defined as $D_{i}$.

(4) Peripheral nodes of a cell: All of the nodes surround the central node $N_{i}$ are the peripheral nodes of cell $D_{i}$. It can be seen that the peripheral nodes of cell $D_{i}$ are the neighbor nodes of the central node $N_{i}$.

(5) The embedded elements of a cell: The neighbor elements of central node $N_{i}$ are called embedded elements of cell $D_{i}$.

The attribute of cell $D_{i}$ are given as follows:

(1) The neighbor nodes of cell $D_{i}$ are fixed and their displacements are zero.

(2) The displacement constraint conditions of central node $N_{i}$ are consistent with the original structure.

Fig.2 is a schematic diagram of cell $D_{i}$. Where $\Delta F_{i}$ is a force increment which is acting on node $N_{i}$ and $\Delta u_{i}$ is the displacement increment caused by $\Delta F_{i}$.

For a discrete structure, the total numbers of cells are equal to the total numbers of nodes.

For cell $D_{i}$, the nodal force $\boldsymbol{F}_{i}$ and the constraint conditions of neighbor nodes $N_{i}^{j}$ determine the displacement of node $N_{i}$. The relationship between the force and the displacement can be described as follows:

(1) Node $N_{i}$ will generate a displacement increment $\triangle \boldsymbol{u}_{i}$ under the action of the nodal force increment $\Delta \boldsymbol{F}_{i}$.

(2) The displacement increment $\Delta \boldsymbol{u}_{i}$ will cause the force increments $\Delta \boldsymbol{F}_{i}^{k}$ of neighbor nodes, $k=1, \ldots, n_{i}$.

The chain of "force $\rightarrow$ displacement $\rightarrow$ force $\rightarrow$..." transfers among all the cells in the structure. Finally, the increment $\triangle \boldsymbol{u}_{i}$ and $\triangle \boldsymbol{F}_{i}{ }^{k}$ approach zero and the structure reaches a balanced state. 


\section{Basic Algorithm}

The basic algorithm of the cellular element method is as follows:

(1) Divide the structure into $e$ elements and $n$ nodes in the same way as the finite element method and then set up the information of geometric topology, material parameters, boundary conditions of forces and displacements, and the convergence error $\varepsilon$. The iterative pointer $\mathrm{k}=0$ and the node's initial displacement value $\boldsymbol{u}_{i}{ }^{0}=0$.

(2) Set up the iterative pointer as $\mathrm{k}=\mathrm{k}+1$. Iterative calculations are done in terms of nodes $\mathrm{N}_{\mathrm{i}}$ $(\mathrm{i}=1,2 \ldots \mathrm{n})$ from step 3 to step 7.

(3) Calculate the displacement increment $\triangle \boldsymbol{u}_{i}$ caused by node unbalanced force $\triangle \boldsymbol{F}_{i}$.

(4) Calculate the force increments $\triangle \boldsymbol{F}_{i}^{j}$ of the neighbor nodes caused by $\triangle \boldsymbol{u}_{i}$.

(5) Calculate $\boldsymbol{u}_{i}^{\mathrm{k}+1}=\boldsymbol{u}_{i}^{\mathrm{k}}+\triangle \boldsymbol{u}_{i}, \quad \boldsymbol{F}_{i}^{j(k+1)}=\boldsymbol{F}_{i}^{j(k)}+\triangle \boldsymbol{F}_{i}^{j}$.

(6) At the end of each iteration, if the maximum relative error of the node displacement $\max \mid\left(\boldsymbol{u}_{i}^{\mathrm{k}+1}\right.$ -

$\left.\boldsymbol{u}_{i}{ }^{\mathrm{k}}\right) / \boldsymbol{u}_{i}{ }^{\mathrm{k}}<\varepsilon$, the iteration will finish and move to the next step. If this condition is not satisfied, then go back to Step 2.

(7) Work out the stress and strain of the elements and nodes by nodal displacements and geometrical physical equations, store the results and end calculation.

The cellular element method adopts the discrete and interpolation techniques which are used in the finite element method. The formation of the element stiffness matrices and the global stiffness matrices of cells are consistent with the finite element method.

Unlike the finite element method, the solution of the equilibrium equation is limited to a single cell in the cellular element method. It only needs to assemble and store the global stiffness matrix of a cell instead of assembling and storing the global stiffness matrix of the whole structure. The order of solving equations is R-order (up to 6th order). Since it is not necessary to form the global equilibrium equation of the structure, the storage problems of large arrays such as the global stiffness (quality) matrices are not necessary. This method requires low computer hardware and the algorithm is simple and it is easy to the compile general program. However, the computing time is too long, especially when the model has a large number of nodes and elements. Therefore, it is necessary to improve it.

\section{Improved Algorithm}

Analyzing the basic algorithm of the cellular element method, the factors that affect the computing speed and times are as follows:

(1) The number of iterative calculations. The ending of the calculation depends on whether the maximum relative errors of nodal displacements reach a predetermined accuracy. The more number of iterations required, the more time that is needed.

(2) The solution of the cell interior. In each iterative calculation, some work is needed for each cell $D_{i}$, including calculating the displacement increment $\triangle \boldsymbol{u}_{i}$ of the central node $N_{i}$ under the nodal force increment $\Delta \boldsymbol{F}_{i}$, and the nodal force increments $\triangle \boldsymbol{F}_{i}^{j}$ of neighbor nodes caused by $\triangle \boldsymbol{u}_{i}$. In fact, it is equivalent to calculating a small structure.

For the first factor, the number of iterations is related to element characteristics. Therefore, it is necessary to study new type elements to reduce the number of iterations. For the other factor, the solution of cell interior is similar to calculating a small structure (cellular level). Though the time is short for calculating one cell, the total time is long because the numbers of cells are large and each circulation requires calculation. The relationship among $\Delta \boldsymbol{F}_{i}, \Delta \boldsymbol{u}_{i}$ and $\Delta \boldsymbol{F}_{i}^{j}$ requires a solution for different $\Delta \boldsymbol{F}_{i}$ in every circulation. In fact, they have a certain ratio which is unrelated to $\Delta \boldsymbol{F}_{i}$ in linear-elastic analysis when the material and size are given. Therefore, what could be considered is to store the ratio in the first circulation and distribute it to the remaining circulations. In this way, it is not necessary to solve equilibrium equations in each circulation and it would save more time. According to this idea, the improved algorithm is:

(1) Divide the structure into $e$ elements and $n$ nodes in the same way as the finite element method, and then set up the information of geometric topology ,material parameters, boundary conditions of 
forces and displacements, and convergence error $\varepsilon$. The iterative pointer $\mathrm{k}=0$ and node's initial displacement value $\boldsymbol{u}_{i}{ }^{0}=0$.

(2)Set up the iterative pointer as $\mathrm{k}=1$. Iterative calculations are done in terms of nodes $N_{i}(\mathrm{i}=1,2 \ldots \mathrm{n})$ from step 3 to step 6.

(3) Calculate the displacement increment $\triangle \boldsymbol{u}_{i}$ caused by node unbalanced force $\triangle \boldsymbol{F}_{i}$.

(4) Calculate the force increments $\triangle \boldsymbol{F}_{i}{ }^{j}$ of the neighbor nodes caused by $\triangle \boldsymbol{u}_{i}$.

(5) Solve the ratio among $\Delta \boldsymbol{F}_{i}, \triangle \boldsymbol{u}_{i}$ and $\Delta \boldsymbol{F}_{i}^{j}$ and store it in the array $\mathrm{K}$.

(6) Calculate $\boldsymbol{u}_{i}^{\mathrm{k}+1}=\boldsymbol{u}_{i}^{\mathrm{k}}+\triangle \boldsymbol{u}_{i}, \quad \boldsymbol{F}_{i}^{j(k+1)}=\boldsymbol{F}_{i}^{j(k)}+\triangle \boldsymbol{F}_{i}^{j}$.

(7)Set up the iterative pointer as $\mathrm{k}=\mathrm{k}+1$.Iterative calculations are done in terms of nodes $N_{i}$ $(\mathrm{i}=1,2 \ldots \mathrm{n})$ from step 8 to step 11.

(8)Calculate the displacement increment $\triangle \boldsymbol{u}_{i}$ and the force increments $\triangle \boldsymbol{F}_{i}^{j}$ of the neighbor nodes by the node unbalanced force $\triangle \boldsymbol{F}_{i}$ and the ratio in the array $\mathrm{K}$.

(9) Calculate $\boldsymbol{u}_{i}^{\mathrm{k}+1}=\boldsymbol{u}_{i}^{\mathrm{k}}+\triangle \boldsymbol{u}_{i}, \quad \boldsymbol{F}_{i}^{j(k+1)}=\boldsymbol{F}_{i}^{j(k)}+\triangle \boldsymbol{F}_{i}^{j}$.

(10) At the end of each iteration, if the maximum relative error of node displacement $\max \mid\left(\boldsymbol{u}_{i}^{\mathrm{k}+1}\right.$ $\left.\boldsymbol{u}_{i}{ }^{\mathrm{k}}\right) / \boldsymbol{u}_{i}{ }^{\mathrm{k}} \mid<\varepsilon$, the iteration will finish and move to the next step. If this condition is not satisfied, then go back to Step 7 .

(11) Work out the stress and strain of the elements and nodes by node displacement and geometrical physical equation, store the results and end calculation.

\section{Contrast Test}

In order to know the effect of the improved algorithm, a simple model was analyzed as an example.

(1) Calculation model

A cantilever rectangular plate was fixed on the left side, with $5 \mathrm{~m}$ length, $3 \mathrm{~m}$ height and $0.02 \mathrm{~m}$ of thickness. The young modulus $\mathrm{E}=210 \mathrm{GPa}$ and Poisson ratio $\mathrm{V}=0.3$. A concentrated load $(\mathrm{P}=6000 \mathrm{kN})$ was applied at the upper right corner of the plate. Regardless of weight, the deformation and stress were calculated.

In order to compare the computing time of the two algorithms, 3-node triangular elements were used to divide the structure with maximum edge lengths of $1 \mathrm{~m}, 0.5 \mathrm{~m}, 0.25 \mathrm{~m}, 0.125 \mathrm{~m}$ and $0.0625 \mathrm{~m}$ respectively. (Shown in Fig.3)
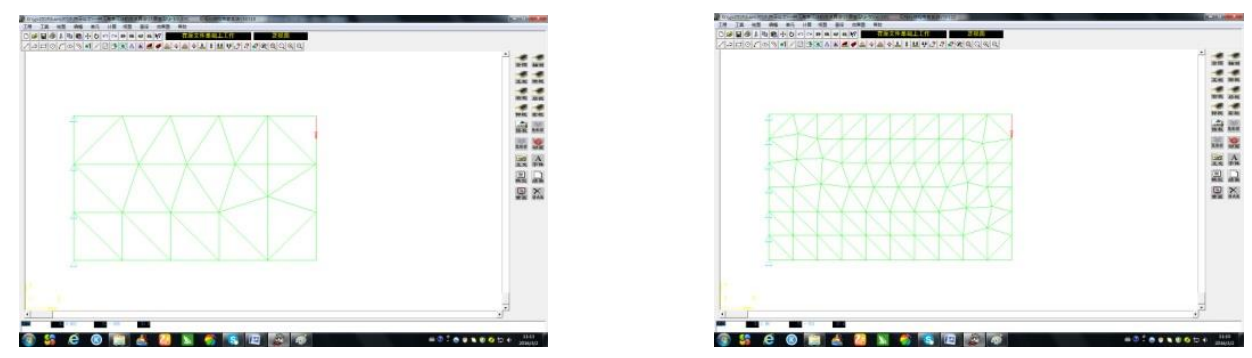

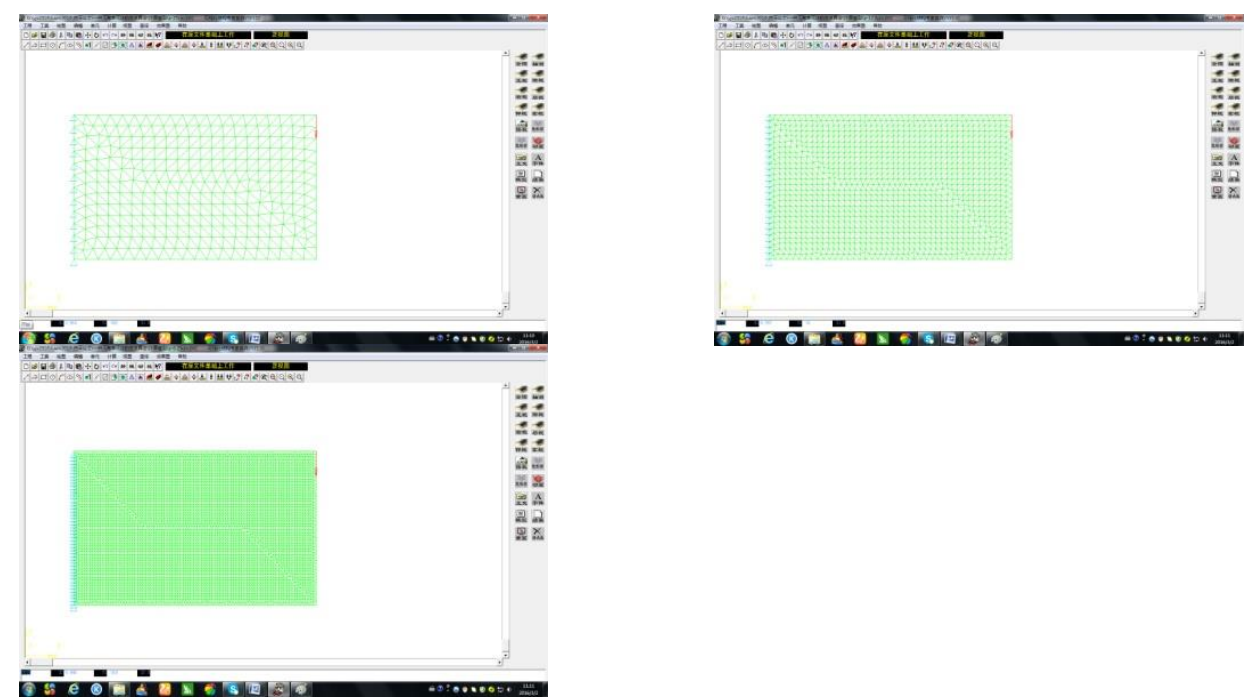

(2) Computing platform

Fig.3 The discrete model with different mesh density

Computer: PC, Intel(R)Core(TM)i5-3470,CPU@3.2GHz.

Software (self-developed software): VSES(Visual Structure Expert System).

(3) Calculation results

Setting up the maximum relative error $\varepsilon=0.01$, the calculation results of each model are shown in Table 1.

Table 1 The calculation time of each model (s)

\begin{tabular}{cccccc}
\hline The maximum length of element & 1 & 0.5 & 0.25 & 0.125 & 0.0625 \\
\hline m $)$ & 53 & 196 & 845 & 3685 & 15089 \\
Numbers of element & 24 & 78 & 305 & 1273 & 5117 \\
Numbers of nodes & $<1$ & $<1$ & $<1$ & 2.6 & 39 \\
Finite element method & 2 & 9 & 37 & 114 & 419 \\
Basic algorithm & 1 & 6 & 24 & 72 & 256 \\
Improved algorithm & & & &
\end{tabular}

Table 1 shows:

(1) In each model, the computing time of the basic algorithm was longer than that of the finite element method. Also, the more elements and nodes used, the greater the difference was between the two. Studying the causes, computing time of the finite element method was mainly spent on solving the global equilibrium equations. Good progress has been made in the technique for fast solving of large linear equations. Used herein is the triangular decomposition method (LDLT), which is a fast solving linear equations method. Therefore, the computing speed of finite element method has reached the ideal level. However, the computing time of the cellular element method was mainly spent on the solution of cell interior and multiple circulations. Research in this area has yet to be a breakthrough. From the current point of view, compared with the finite element method, the cellular element method doesn't have any special requirement on the computer memory but has a high requirement on the computing speed. It is a measure of time for space. Of course, it should be noted that the cellular element method is easier to achieve parallel computing than the finite element method.

(2) In each model, the improved algorithm in this paper had higher speed than the basic algorithm. It took less time, and the more elements and nodes used, the greater the difference was between the two. The main reason was that a new technique was used in the solution of cell interior.

Conclusions

The cellular element method is a promising method for computing, which may be an alternative measure to the finite element method in some respects, especially in parallel computing. But it is still in its infancy, there are many issues that need to be studied and solved. In this paper, an 
improved algorithm was proposed to improve the computing speed and the speed was improved significantly compared with the basic cellular element method. It should be noted that although this improvement has some effect, it still takes a long time. Further research is needed to accelerate the computing speed especially in the speed of convergence.

\section{References}

[1] SHEN Chengwu, TANG Xiaobing, YANG Jixin. Approach to Mechanical Analysis of Plane Truss Using Cellular Automata[J]. Journal of Wuhan Transportation University, Vol.24 No.2(2000),p.105 108

[2] SHEN Chengwu, DAI Shiliang, YANG Jixin, TANG Xiaobing. Cellular Automata For Analysis of Plane Problems in Theory of Elasticity[J]. Tsinghua Univ (Sci\& Tech). Vol.41 No.11(2001),p.35 38

[3] YANG Jixin, WANG Cheng, SHEN Chengwu. Cellular Element Method[J]. Journal of Solid Mechanics. Vol.25 No.2(2003),p.203 207

[4] YANG Jixin. Parallel cellular element method [J]. Chinese Journal of Computational Mechanics. Vol.23 No.1(2006),p.97 100

[5]YANG Jixin, ZHANG Baokui, ZENG Yan. From Moment Distributed Method to Cellular Element Method[J]. Journal of Wuhan University of Technology. Vol.28 No.6(2004),p.35 38 2004.6: $35 \sim 38$ 